\title{
A case of infectious mononucleosis in a patient with ulcerative colitis on infliximab
}

\author{
Angela C. Bien, Kasiah Banks, J ill K. J. Gaidos, J ohn F. Kuemmerle \\ Gastroenterology, Hepatology, and Nutrition, Virginia Commonwealth University, Richmond, USA.
}

Correspondence: Angela C. Bien. Address: 1200 E. Broad St., West Hospital 14th floor, P.O. Box 980341, Richmond, VA 23298-0341, USA. Email: acbien@mcvh-vcu.edu

Received: February 26, 2014

DOI : $10.5430 /$ crim.v1n2p202

Online Published: July 3, 2014

URL: http://dx.doi.org/10.5430/crim.v1n2p202

\section{Abstract}

A 19 year old woman with ulcerative colitis presented to our clinic with low grade fevers, fatigue, nausea, dizziness, anorexia, and swollen neck glands. Her symptoms developed after receiving induction and two maintenance doses of infliximab. Her physical exam was notable for bilateral large, tender submandibular lymph nodes. Her laboratory values revealed an elevated C-reactive protein, a normal white blood cell count, but prominent lymphocytes, and a positive heterophile antibody. Tumor necrosis factor (TNF- $\alpha$ ) is a central mediator in chronic inflammation and, thus, has been a target of treatment in chronic inflammatory disorders, such as inflammatory bowel disease (IBD). It also plays an important role in the immune response and host defense, especially in the control of viral infections. Increased rates of bacterial infection in patients treated with anti-TNF- $\alpha$ is a known risk and, recently, there have been investigations into risks of other opportunistic infections. However, few studies have looked at the risk of viral infections and anti-TNF- $\alpha$ use. As a result, there are no (or few) guidelines for treatment of patients who develop a viral infection, such as Epstein-Barr virus, while being treated with an anti-TNF- $\alpha$ agent. Many of the studies assessing the possible association between anti-TNF- $\alpha$ use and viral infections have been case reports. Therefore, in order for physicians to better treat these immune compromised patients, more needs to be dedicated to investigating the risk of viral infections and in turn, how best to monitor, prevent, and treat patients who develop viral infections while on immunosuppressants, such as anti-TNF- $\alpha$ agents.

\section{Keywords}

Inflammatory bowel disease, Infliximab, Infectious mononucleosis, Epstein-Barr virus

\section{I ntroduction}

When reviewing the side effects of immunosuppressives used to treat IBD, it is common for clinicians to mention an increased risk of developing infections. This increased risk is supported by the current available literature, but there is little data to characterize the incidence of opportunistic infections, and even less on the incidence of viral infections with the use of specific IBD treatments. In the following case report, we discuss a patient with ulcerative colitis (UC) who was being treated with infliximab and subsequently was diagnosed with infectious mononucleosis. 


\section{Case presentation}

A 19 year old woman with pan-UC diagnosed one year prior by pathology obtained at endoscopy, presented to our ambulatory gastroenterology clinic with a one week history of low-grade fevers, malaise, anorexia, and submandibular lymphadenopathy. Her UC was not well controlled on mesalamine so she was started on infliximab in November 2011. She had received the induction doses as well as two maintenance doses of infliximab at $5 \mathrm{mg} / \mathrm{kg}$ when the above mentioned symptoms started. In addition, her abdominal pain was unchanged and she was having diarrhea two to three weeks after her infusions.

Her physical exam revealed an afebrile young woman with large, bilateral submandibular lymphadenopathy, noted to be firm, mobile, and tender to palpation. Her abdomen was soft, not distended or tender. Her laboratory evaluation revealed an elevated aspartate aminotransferase of 113 (units/L), elevated alanine aminotransferase of 126 (units/L), elevated alkaline phosphatase of 428 (units/L), elevated C - reactive protein of $2.1(\mathrm{mg} / \mathrm{dL})$, decreased hemoglobin of $9.1(\mathrm{~g} / \mathrm{dL})$, white blood cell count within normal limits at 10.7 10e9/L, with 65.4\% lymphocytes (normal range 21.1\%-52.8\%), and heterophile antibody positive.

Her infliximab infusions were held and she was started on prednisone 40mg. Within 6 weeks, she recovered completely from her infectious mononucleosis and the infliximab infusions were restarted without any complications.

\section{Discussion}

Patients with inflammatory bowel disease (IBD) are at an increased risk of developing infections, which can be characterized as non-therapy dependent, i.e. due to IBD, or therapy-dependent, i.e. due to the medications used to treat IBD $^{[1]}$. It is difficult to determine the incidence of opportunistic infections among IBD patients due to limited reporting. The medications used to induce and/or maintain remission, such as corticosteroids, immunomodulators [Azathioprine (AZA), 6-mercaptopurine (6-MP), and methotrexate], and biologics (infliximab, certolizumab pegol, adalimumab, and natalizumab) increase the risk for infections. A case-control study by Tourner, et al. showed that use of immunesuppressive medications was significantly associated with the development of opportunistic infections in IBD patients and, when used in combination, the risk was further increased ${ }^{[2]}$. This increased risk was also shown in a retrospective cohort study by Marehbian, et al ${ }^{[3]}$. The updated Therapy, Resource, Evaluation, and Assessment Tool (TREAT) registry looked at long-term outcomes of various treatment regimens used in Crohn's disease and found a higher incidence of overall infections with infliximab ${ }^{[4]}$.

The association with immunosuppressive medication use in IBD patients and viral infections is less well documented. Tumor necrosis factor (TNF- $\alpha$ ) plays an important role in the body's response to viral infections. Cells of the innate immune system and cytotoxic $\mathrm{T}$ cells produce cytokines, such as TNF- $\alpha$, which recruit and activate macrophages, natural killer cells, and T cells, triggering an antiviral function in effector T cells ${ }^{[5]}$. TNF- $\alpha$ can also kill viruses independent of the actions of cytotoxic T cells ${ }^{[5]}$. Thus, blocking TNF- $\alpha$ impedes the body's response to viral infections and leads to their reactivation.

In IBD patients receiving infliximab, herpes simplex virus (HSV), cytomegalovirus (CMV), Epstein-Barr virus (EBV) or varicella zoster virus (VZV) virus infections have accounted for the majority of viral infections. EBV, one of the most common human viruses, lays dormant in memory B cells and is reactivated by acquired immunodeficiency ${ }^{[6]}$. There have been several case reports that have depicted associations between EBV-related conditions, such as infectious mononucleosis and lymphoproliferative disorders, and the use of anti-TNF agents ${ }^{[7]}$. However, there are no documented reports of EBV infections specifically in IBD patients treated with anti-TNF agents, such as infliximab. Currently, there are no guidelines regarding discontinuing immunosuppressives at the onset of EBV infections, if anti-viral treatment needs to be initiated at the time of diagnosis, or if patients need prophylactic therapy when immunosuppressive agents are 
started. In patients treated with anti-TNF agents who develop lymphoproliferative disorders, regression of disease occurs upon medication cessation ${ }^{[8]}$. Therefore, pending further investigation, IBD patients should receive preventative vaccinations, when possible, prior to initiating treatment with immunosuppressive agents, such as infliximab, and require close monitoring while immune suppressed for potential bacterial, fungal, and viral infections.

\section{References}

[1] Epple H-J. Therapy- and non- therapy-dependent infectious complications in inflammatory bowel disease. Dig Dis. 2009; 27: 555-9. http://dx.doi.org/10.1159/000233297

[2] Tourner M, Loftus EV Jr, Harmsen WS. Risk factors for opportunistic infections in patients with inflammatory bowel disease. Gastroenterology. 2008; 134: 929-36. http://dx.doi.org/10.1053/j.gastro.2008.01.012

[3] Marehbian J, Arrighi HM, Hass S, et al. Adverse events associated with common therapy regimens for moderate-to-severe Crohn's disease. Am J Gastroenterol. 2009; 104: 2425-33. http://dx.doi.org/10.1038/ajg.2009.322

[4] Lichtenstein GR, Feagan BG, Cohen RD, et al. Serious infection and mortality in patients with Crohn's disease: More than 5 years follow-up in the TREAT registry. Am J Gastroenterol. 2012; 107: 1409-22. http://dx.doi.org/10.1038/ajg.2012.218

[5] Guidotti LG, Chisari FV. Noncytolytic control of viral infections by the innate and adaptive immune response. Annu Rev Immunol. 2001; 19: 65-91.

[6] Centers for Diseases Control and Prevention. Epstein-Barr virus and infectious mononucleosis. Available from: http://www.cdc.gov/ncidod/diseases/ebv.htm (2006).

[7] Sari I, Birlik M, Akar S, Onen F, Kargi A, Akkoc N. Atypical infectious mononucleosis in a patient receiving tumor necrosis factor alpha inhibitory treatment. Rheumatol Int. 2009; 29: 825-6. http://dx.doi.org/10.1007/s00296-008-0775-5

[8] Shale M, Kanfer E, Panaccione R, Ghosh S. Hepatosplenic T-cell lymphoma in inflammatory bowel disease. Gut. 2008; 57: 1639-41. http://dx.doi.org/10.1136/gut.2008.163279 